\title{
Los GRDs Psiquiátricos: Una investigación pendiente
}

A. Seva-Díaz*

A. Seva-Fernández ${ }^{* *}$

* Catedrático de Psiquiatría y Psicología

Médica, Jefe del Departamento

de Psiquiatría, Facultad de Medicina

y Zaragoza Clínico Universitario, Zaragoza

** Médico Especialista en Medicina Familiar

y Comunitaria, Zaragoza

ESPAÑA

\begin{abstract}
RESUMEN - Los GRDs (grupos relacionados en el diagnóstico) se han mostrado muy útiles para lograr aproximaciones a los costes generados por la actividad médica con referencia a los diferentes diagnósticos, sin embargo en Psiquiatría las categorías diagnósticas actualmente utilizadas son tan limitadas que requieren una mayor profundización y discernimiento.

Los autores estudian los 10.974 ingresos psiquiátricos producidos entre los años 1975 al 2000 en el Departamento de Psiquiatría del Hospital Clínico Universitario de Zaragoza (España), mostrando las diferencias en cuanto a las estancias medias según diferentes factores como el sexo de pertenencia, la edad, el estado civil, la situación laboral, la clase social, el lugar de residencia, la dependencia asistencial del paciente, el tipo de ingreso, la tasa de reingresos y por supuesto el diagnóstico psiquiátrico.
\end{abstract}

\section{Introducción: El producto hospitalario y su medida. Los GRDs en Psiquiatría}

Con el fin de mejorar la gestión y la eficiencia de los Servicios, surgieron hace años los sistemas de medición del case-mix que intentaron crear agrupaciones de pacientes unidos por algunos aspectos que los homogenizan. Y así surgieron los Patient Manage- ment Categories o PMC, el Patient Severity Index o PSI, el Disease Staging o DS, el Acute Physiology and Chronic Health Evaluation o APACHE, el Age System State Complications Response o AS-SCORE, los Ambulatory Visit Groups o AVG y los Grupos Relacionados con el Diagnóstico o $G R D$, entre otros.

Un grupo de investigadores americanos de la Universidad de Yale dirigidos por Fetter y Thompson, consiguieron diseñar este sistema 
de consumo de recursos a principios de los años setenta, siendo adoptado por la Administración americana en el año 1983 a fin de realizar los pagos por proceso. De esta manera y a partir de 700.000 altas hospitalarias analizadas, se consiguió clasificar a los pacientes en 383 GRDs de acuerdo con la ICD-8. Posteriormente fue adaptado a la ICD-9 para lo cual se estudiaron cerca de millón y medio de altas hospitalarias apareciendo entonces 470 GRDs. Como el número de grupos era muy elevado, se consiguió diseñar 23 Categorías Diagnósticas Mayores o CDM, dentro de las cuales solo una, la 19, hacía mención explícita a Trastornos mentales, aunque otras dos mas podían contener a pacientes nuestros, como sucede con la 1 Enfermedades y trastornos del sistema nervioso y la 20 Uso de drogas y trastornos mentales inducidos por drogas. Estas CDM se concibieron para que se correspondieran con un aparato o con un sistema orgánico principal y no necesariamente con una etiología. Como no todas las enfermedades pudieron relacionarse con un determinado y exclusivo sistema o aparato orgánico, hubo de crearse algunas CDMs residuales para las enfermedades infecciosas sistémicas, para las mieloproliferativas, etc.).

Por otro lado, se estudió la influencia que patologías asociadas o comórbidas, complicaciones, o simplemente la edad de los pacientes pudieran influir en el consumo de recursos hospitalarios. Así se acordó considerar como complicación o comorbilidad importante, a aquella que asociada a un diagnóstico principal prolongase al menos en un día, la estancia en el hospital en mas del $75 \%$ de los casos.

Para conocer la complejidad de la casuística existen dos indicadores: el Indice Case-mix y el Peso Relativo del Centro. El Indice Casemix es la razón entre la estancia media ajustada por funcionamiento y la estancia media del estándar, de manera que cuando éste es supe- rior a 1, quiere ello decir que la casuística atendida por ese Servicio es de mayor complejidad que la del estándar. El Peso Relativo del Centro es la razón entre el peso medio del hospital y el propio del estándar, de modo que un valor superior a 1 nos indica la existencia de una casuística mas costosa.

Para calcular la eficiencia en la gestión de las camas, contamos con otros dos índices: el llamado Índice Funcional y la Razón de Funcionamiento Estándar. El Índice Funcional es la razón entre la estancia media ajustada por case-mix y la estancia media del estándar, de manera que si éste es superior a 1 nos indica que ese Servicio posee una menor eficiencia en la gestión de sus camas. La Razón de Funcionamiento Estándar, es la razón entre la estancia media observada en el hospital o servicio y la estancia media esperada si tratara cada GRD con la estancia media del estándar de comparación, de modo que cuando es mayor que 1 , nos indica que dicho hospital necesita un mayor número de días de estancia que el estándar y por tanto es menos eficiente (F. Errasti 1996, M.A. Asenjo 1999, J.L. Temes-Montes et al. 1997).

Los GRDs así se han convertido en útiles herramientas para objetivar los costes producidos por la actividad de los Servicios, constituyendo un sistema de clasificación de episodios de hospitalización, de manera que los pacientes de cada clase, se espera que consuman una cantidad similar de recursos. Sin embargo, los problemas que se detectan en Psiquiatría derivan de una serie de limitaciones que los GRDs tienen, tales como la pobreza de las categorías diagnósticas que manejan, lo que hace que dentro de un mismo grupo como es el de Psicosis (GRD 430) se incluya la mayor parte de la patología psiquiátrica sin tener en cuenta de a qué psicosis nos estamos refiriendo (Esquizofrenias, Psicosis Orgánicas o Exógenas, etc.). Por 
otro lado, es muy difícil calcular la gravedad de cada patología asistida, y por supuesto la diversidad de factores psicosociales puestos en juego en cada paciente, de manera que no es lo mismo que determinada patología psiquiátrica se dé en el seno de una familia en la que exista un buen apoyo económico y/o afectivo, que en otra en la que falten éstos. Y no digamos nada en cuanto a las posibilidades que puedan existir en el entorno del hospital para poder continuar la asistencia de un determinado enfermo en las diferentes instituciones extrahospitalarias (Hospitales de Día, Centros de Día, Centros de Salud, Residencias Psiquiátricas de Media Estancia y de Larga Estancia, Asistencia domiciliaria, etc.). En unos casos el alta será facilitada extraordinariamente, pero en otros casos se dará una prolongación mayor de ésta. Otro hecho igualmente muy importante hace referencia a la calidad del alta producida por determinado Servicio, aspecto éste que escapa en la evaluación que los GRDs hacen. Así resulta que los GRD, en el caso de nuestra especialidad, solo explican el 5,6\% de la varianza en los tiempos de estancia como English y colabs. en 1986 advirtieron. Así que otros autores como Mezzich y Sharfstein han recomendado incluir índices de gravedad y psicosociales (V. Kovess y D. Soyris 1994, R.F. Averill et al. 1995, M. Casas 1995).

Se dan grandes deficiencias, en cuanto a la aplicación que hace el Instituto Nacional de la Salud (INSALUD) de los GRDs en nuestra especialidad.

Así de los 474 casos de hospitalización producidos en el año 2000, nada menos que 248 pertenecen a ese cajón de sastre de las Psicosis. La estancia media, en este caso, se sitúa en 16,2 días y la depurada (que excluye los casos extremos) en 13,2 días. El peso relativo del proceso Psicosis es de 1,5274, lo cual nos indica un nivel de complejidad de la patología asistida en este caso impor- tante, lo que justifica en parte las estancias producidas, pero que no corresponden a nuestro país sino a los pesos relativos del Medicare/New York del GRD.

En segundo lugar, en orden de frecuencia, aparecen los Trastornos de Personalidad y el obsoleto diagnóstico de Neurosis diferentes a la Neurosis depresiva en tercer lugar. El primero consta de 30 casos y aparece con una estancia media de 13,6 días y depurada de 10,4 días. Su peso relativo es bajo, de solo 0,6880. Las Neurosis diferentes a la Neurosis depresiva aparece con 27 casos e hicieron una estancia media de 13,9 días, siendo la depurada de 9,3 días. Su peso relativo es de 1,0537 por lo que puede considerarse con un nivel de complejidad mínimo. En cuarto lugar, tenemos con 21 casos a las Neurosis depresivas con una estancia media de 10,1 días y una depurada de 9,2 días. En quinto lugar aparecen los Abusos/Dependencia a alcohol que dan una estancia media de 12,7 días y una depurada de 11,3 días. En sexto lugar estan las Reacciones Agudas/Desadaptaciones/Disfunción psicosocial, que ofrecen una estancia media de 13,1 días y una estancia media depurada de 7,7 días. La llamada aquí Rehabilitación de trastornos compulsivos de la nutrición presenta la estancia media más elevada con 43,3 días la bruta y con 39 días la depurada. Su peso relativo es muy alto con una cifra de 3,1830 .

Como vemos y según todo esto, la estancia media general del Departamento es de 15,7 días, siendo la estancia media depurada de 12,4 días y nuestro peso relativo que mide la complejidad de los cuadros tratados de 1,2642 .

Estos problemas han preocupado a diferentes autores en estos últimos años (U. Meise y H. Hinterhuber 1998) refieren como desde el año 1997 se implantó en Austria este modelo de los DRGs en la 
financiación de los hospitales. Pues bien, según ellos afirman, la utilización en Psiquiatría de este sistema sin adaptarlo a la idiosincrasia de nuestros pacientes puede ser muy perjudicial en la asistencia futura de los mismos. A similares conclusiones llegaron autores australianos como D. BenTovin et al. $(1994,1996)$ hace pocos años.

Entre otros aspectos a considerar, y a fin de evaluar adecuadamente los DRGs psiquiátricos será necesario recoger meticulosamente los diagnósticos secundarios, las complicaciones y en definitiva la patología asociada en cada caso. Así R.J. Goldberg et al. (1996) de un total de 100 pacientes estudiados, en 25 se encontraron 33 complicaciones psiquiátricas y comorbilidades. Pues bien, de esas 33 complicaciones únicamente los médicos habían reflejado 9 (24 por 100) en la hoja del Alta. Naturalmente que si toda la información sobre comorbilidad no figura en el momento del Alta y reflejada en el correspondiente Informe, no será posible evaluar el nivel de complejidad de la patología asistida ni justificar el número de días de estancia, de consumos de fármacos o de procedimientos diagnósticos utilizados (C. Stoskopf et al. 1992).

Otros autores como B. Boot et al. (1997) defienden la adicción de escalas de discapacidad a los DRGs con el fin de poder incluso predecir los días de estancia que determinada patología requerirá.

En fin, otros autores como C.E. Hunter $e t$ al. (1994) y R.D. Goldney et al. (1996) en Australia y S.R. Pirzada et al. (1997) se han ocupado de estos problemas.

\section{Material y método}

Desde hace 26 años venimos coleccionando toda una serie de datos referentes a los ingresos psiquiátricos que han tenido lugar en el Servicio de Agudos del Departamento de Psiquiatría del Hospital Clínico Universitario de Zaragoza (España). Un total de 10.974 ingresos se realizaron entre los años 1975 al 2000. De ellos el 51 por 100 fueron hombres y el 49 por 100 mujeres. Durante la mayor parte de estos años la patología psiquiátrica aguda correspondiente a la provincia de Zaragoza ( $842.419 \mathrm{~h}$ ) fue atendida por este Servicio y a partir de 1992 y de 1994, nuevos Servicios de Agudos se abrieron en tres hospitales generales más.

Como en el año 1993 empezamos a utilizar los diagnósticos los diagnósticos ICD10, abandonando los ICD-9, hemos integrado a la totalidad de los diagnósticos en 22 clases con el fin de poder tratarlos como un conjunto único.

Con el fin de conocer mejor la importancia de algunos factores que pudieran explicar las diferencias en cuanto a las estancias medias a fin de perfeccionar la futura construcción de los GRDs psiquiátricos, hemos estudiado estos 10.974 ingresos atendiendo a factores como el sexo de pertenencia, la edad, el estado civil, la situación laboral, la clase social, el lugar de residencia, la dependencia asistencial del paciente, el tipo de ingreso, la tasa de reingresos y por supuesto el diagnóstico psiquiátrico.

\section{Resultados}

Vemos así en la figura 1 como durante estos años, desde 1975 a 2000 se produjeron 10.974 ingresos de los que el $51 \%$ fueron hombres y el $49 \%$ fueron mujeres.

El estado civil de los mismos fue de un $47 \%$ de solteros, un $43,4 \%$ de casados, un 
$5 \%$ de viudos, un 4,5\% de separados o divorciados y un $0,1 \%$ de religiosos (figura 2 ).

La situación laboral de los mismos (figura 3).

Era de inactivos el $70 \%$ (teniendo en cuenta que la mayor parte de ellos son amas de casa), de activos por cuenta ajena el $26,9 \%$, de activos por cuenta propia el $2,5 \%$, y en situación de marginación el $0,6 \%$. La clase social de estos pacientes era la de alta y media alta en el $1,8 \%$, la de media en el $74,4 \%$, la de mediabaja en el $16,5 \%$, la de baja en el $5,1 \%$, y la de muy baja y marginal en el 2,3\% (figura 4 ).

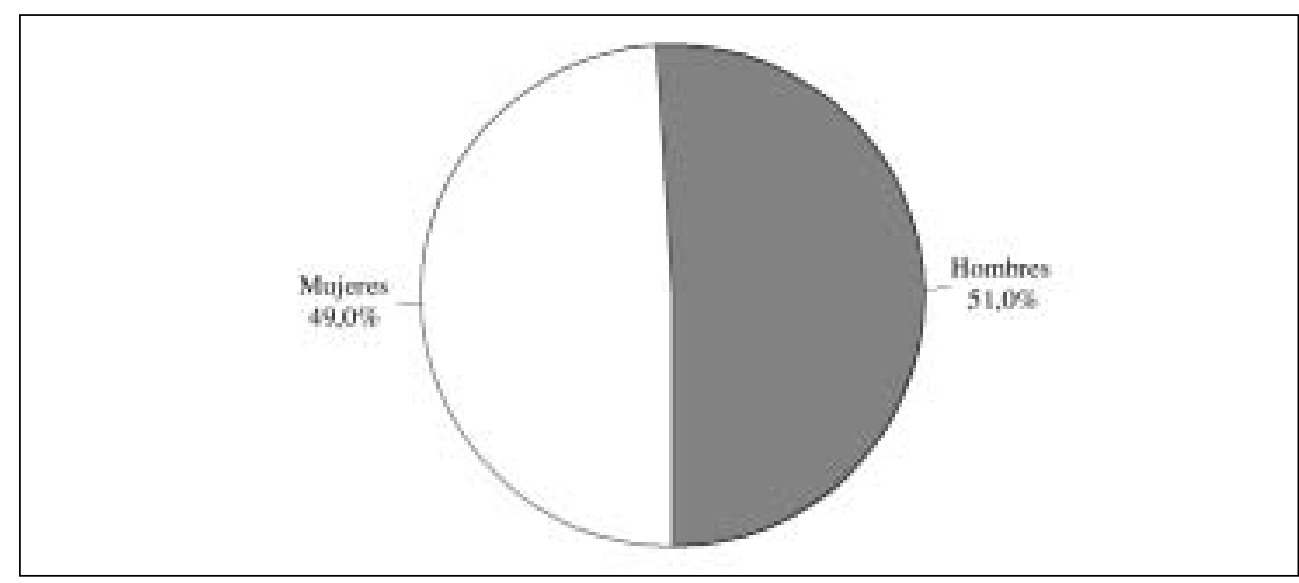

Figura 1. Sexo de los pacientes ingresados desde 1975 a 2000 (26 años).

Departamento de Psiquiatría (Prof. A. Seva). Hospital Clínico Universitario. Zaragoza.

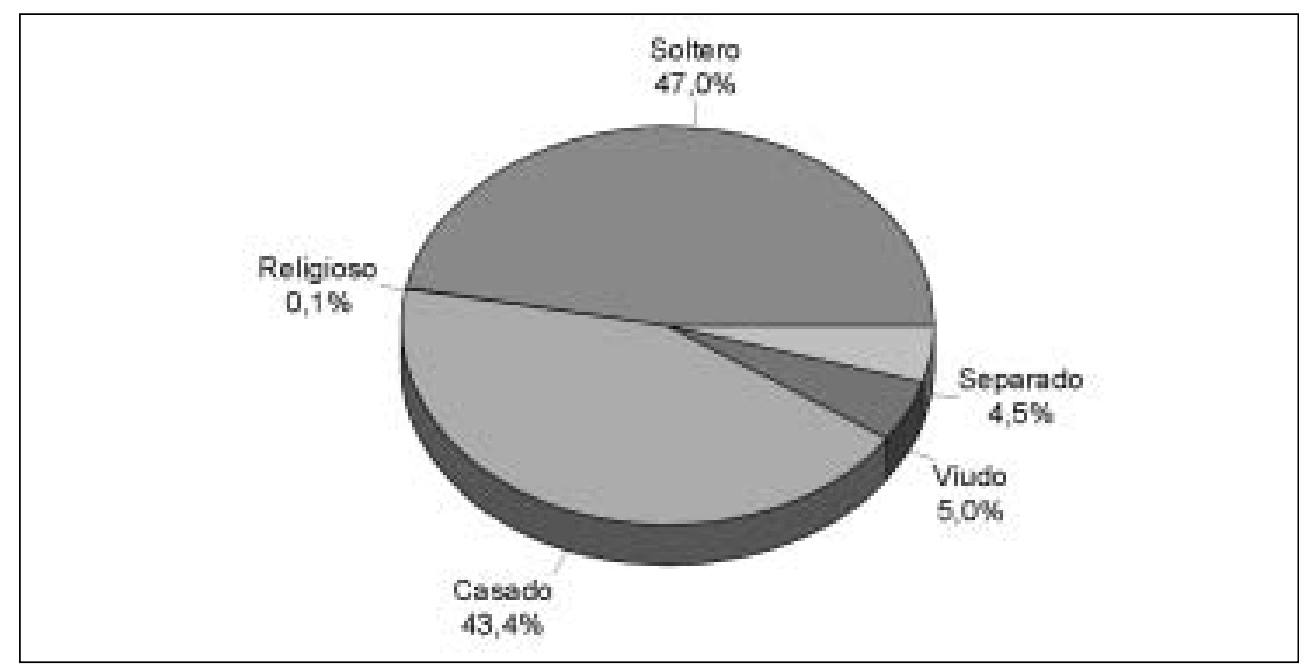

Figura 2. Estado civil de los pacientes ingresados desde 1975 a 2000 (26 años).

Departamento de Psiquiatría (Prof. A. Seva). Hospital Clínico Universitario. Zaragoza. 


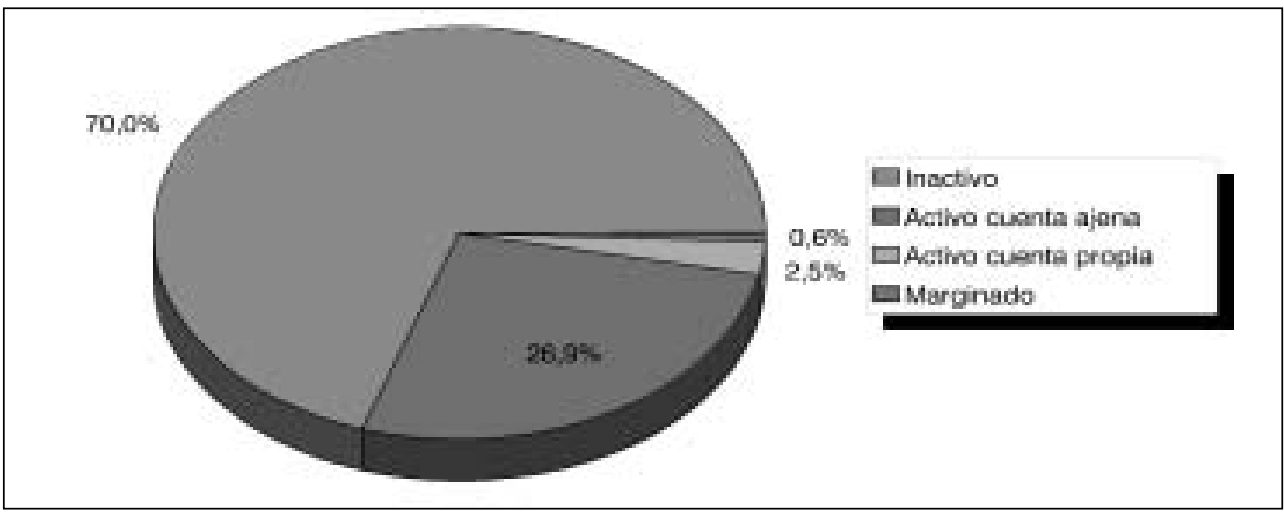

Figura 3. Profesión de los pacientes ingresados desde 1975 a 2000 (26 años). Departamento de Psiquiatría (Prof. A. Seva). Hospital Clínico Universitario. Zaragoza.

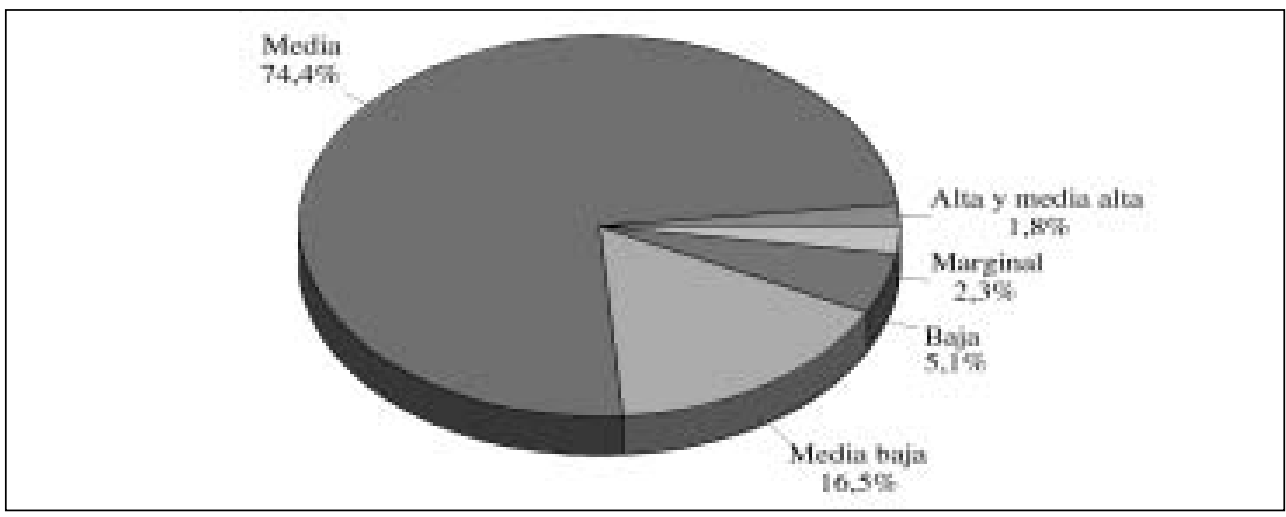

Figura 4. Clase Social de los pacientes ingresados desde 1975 a 2000 (26 años).

Departamento de Psiquiatría (Prof. A. Seva). Hospital Clínico Universitario. Zaragoza.

El lugar de residencia de los mismos era la provincia de Zaragoza en el $92 \%$ de los casos, la de Huesca en el 1,9\%, la de Teruel en el 2,5\%, la de Soria en el $0,4 \%$, la de Logroño en el $0,5 \%$, la de Navarra en el $0,3 \%$ y la de otras provincias en el $1,9 \%$. Los extranjeros supusieron el 0,4\% (figura 5).

Con respecto a la dependencia asistencial de nuestros enfermos a lo largo de estos 25 años (figura 6) resulta que el 83,2\% pertenecen al INSALUD, el 15,2\% a las llamadas Ramas Especiales de la Seguridad Social, el $0,6 \%$ a los Seguros Privados, el 0,5\% eran pacientes privados, el $0,2 \%$ eran pacientes de la antigua Beneficencia y el 0,3\% restante pertenecían a otras formas de dependencia diversas.

La forma de ingreso producida (figura 7) había sido voluntaria en el $97,63 \%$ de los casos y compulsiva en solo el 1,37\%. Mediante orden judicial se produjo el ingreso en el $1 \%$ restante.

Las edades de los pacientes ingresados aparecen en la figura 8 , de modo que el intervalo que va de los 20 a los 29 años fue 


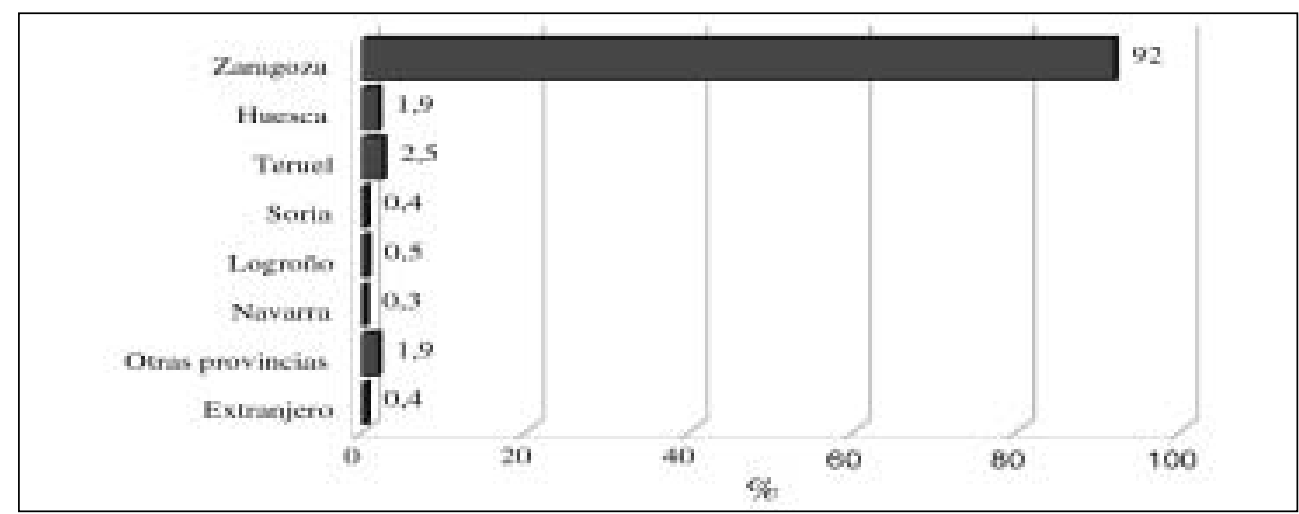

Figura 5. Lugar de Residencia de los pacientes ingresados desde 1975 a 2000 (26 años). Departamento de Psiquiatría (Prof. A. Seva). Hospital Clínico Universitario. Zaragoza.

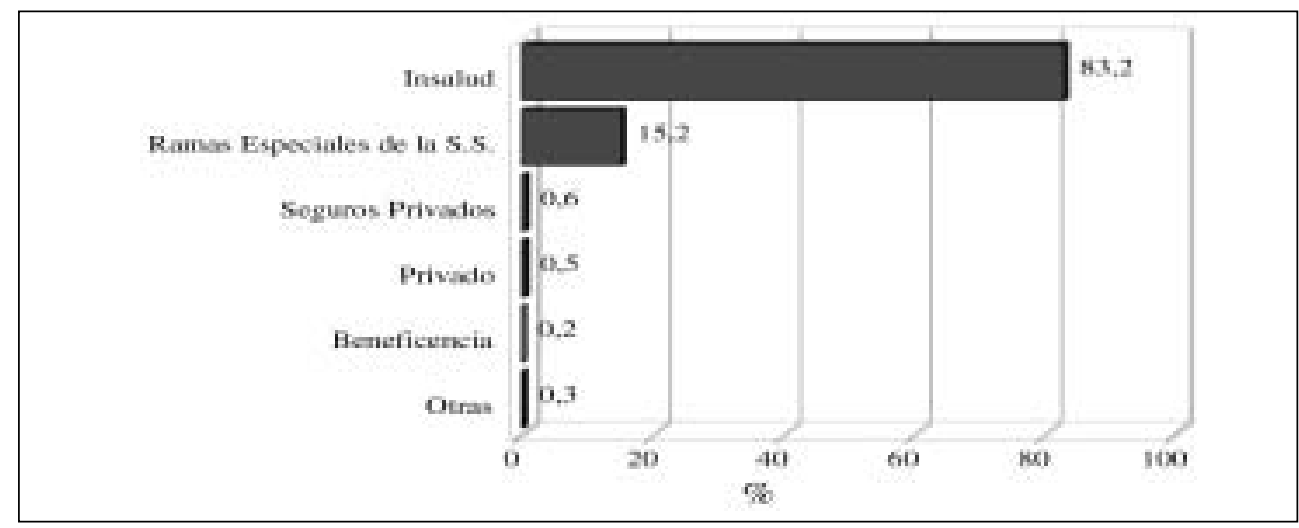

Figura 6. Dependencia Asistencial de los pacientes ingresados desde 1975 a 2000 (26 años). Departamento de Psiquiatría (Prof. A. Seva). Hospital Clínico Universitario. Zaragoza.

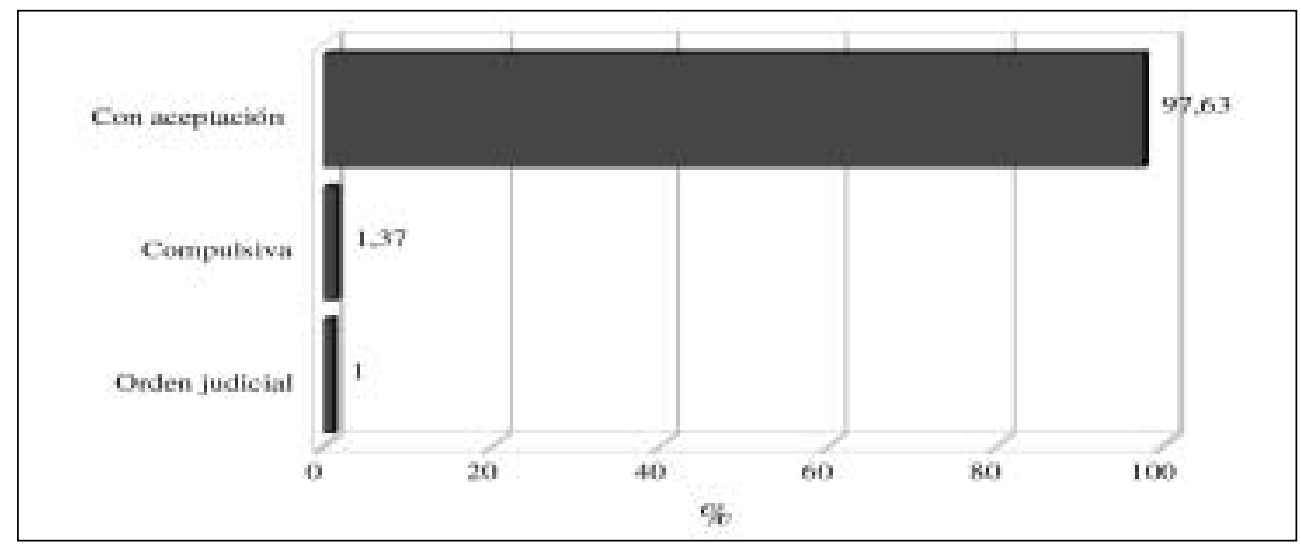

Figura 7. Forma de ingreso de los pacientes ingresados desde 1975 a 2000 (26 años).

Departamento de Psiquiatría (Prof. A. Seva). Hospital Clínico Universitario. Zaragoza. 


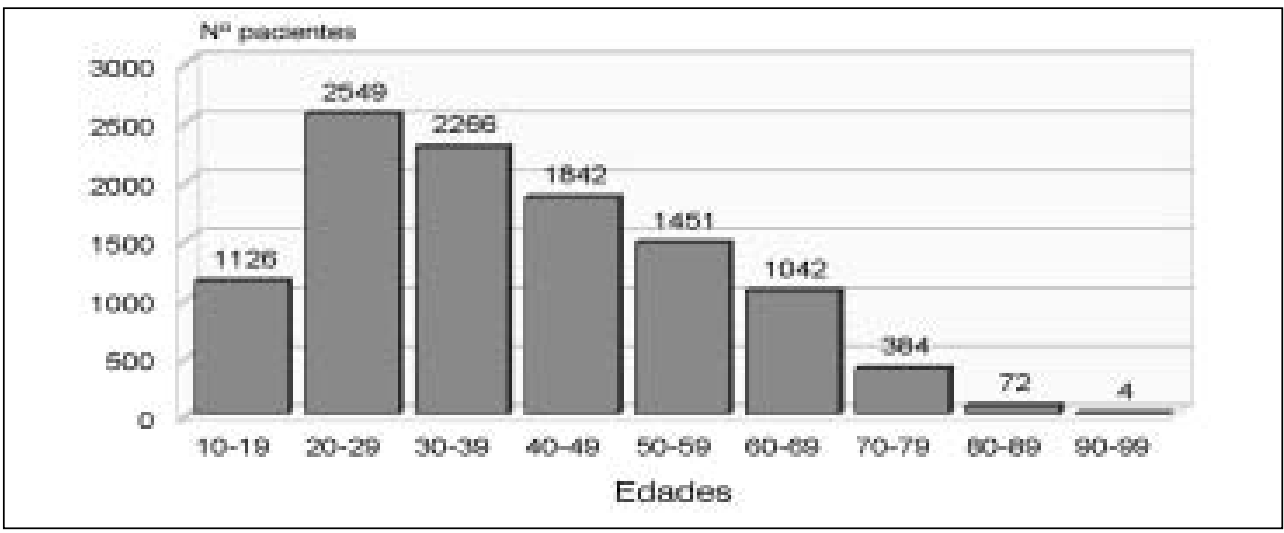

Figura 8. Edades de los pacientes ingresados desde 1975 a 2000 (26 años). Departamento de Psiquiatría (Prof. A. Seva). Hospital Clínico Universitario. Zaragoza.

el grupo más numeroso con 2.549 ingresos, seguido del que va de los 30 a los 39 años con 2.266 pacientes. A continuación figuran los intervalos siguientes desde los 40 a 49 años en adelante, disminuyendo progresivamente su número.
En la figura 9 hemos agrupado los diagnósticos habidos en la totalidad de estos 25 años y ello lo hemos hecho a costa de perder algunos de ellos por la dificultad de encajarlos adecuadamente en los que en esta Figura aparecen. Se aprecia como las Esquizofre-

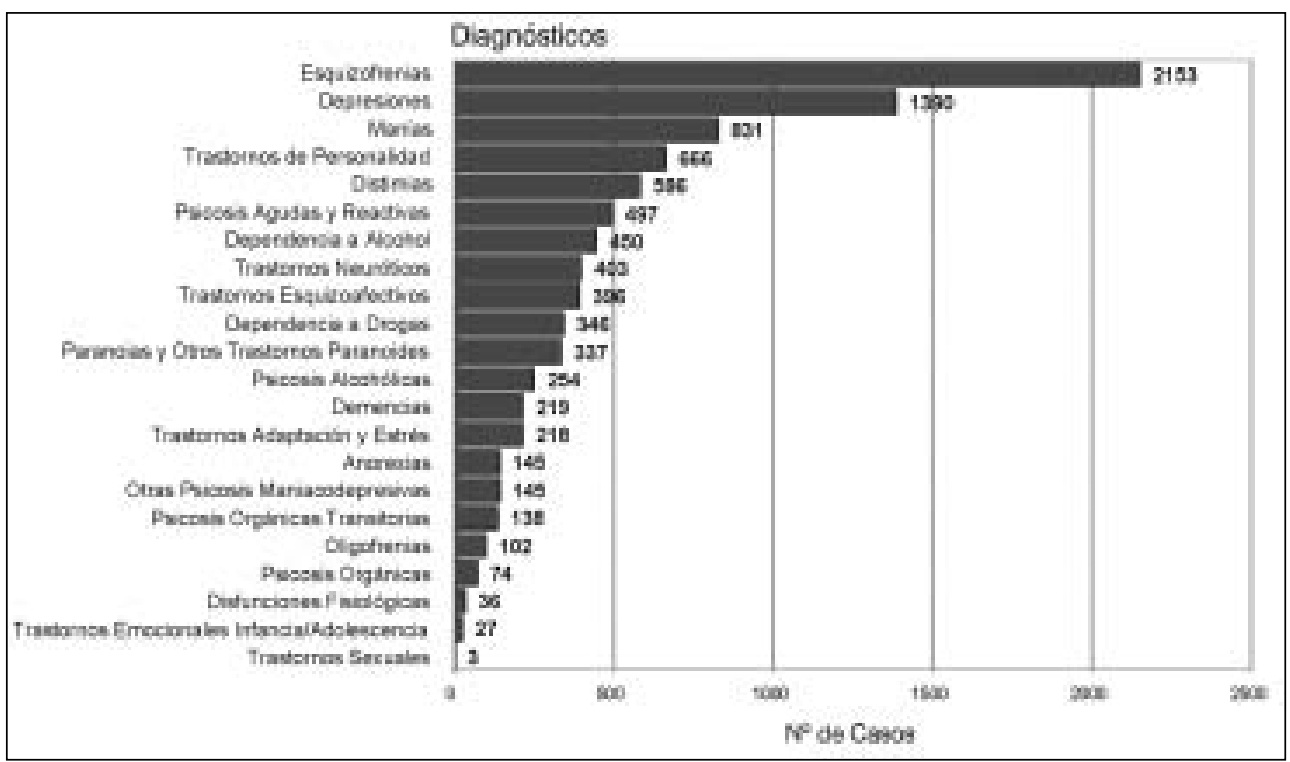

Figura 9. Frecuencia de agrupaciones diagnósticas CIE-10 de pacientes ingresados en 26 años (1975-2000). Departamento de Psiquiatría (Prof. A. Seva). Hospital Clínico Universitario. Zaragoza. 
nias con 2.153 casos representan la mayor frecuencia, siguiendo a ellas las Depresiones con 1.390 casos y a continuación los Cuadros Maníacos con 831 casos, los Trastornos de Personalidad con 655, las Distimias con 586, las Psicosis Agudas y Reactivas con 497, etc.

En la tabla I ofrecemos el número medio de episodios de ingreso según los diferentes diagnósticos, observándose como son las Otras Psicosis Maniacodepresivas y los Tras- tornos Esquizoafectivos con alrededor de 3 a 4 ingresos en estos 26 años, los que mas utilizaron nuestro Servicio de Hospitalización. A estos diagnósticos les siguen las Psicosis Maníacas y las Depresivas con alrededor de 3 episodios de ingreso en estos años.

En la tabla II podemos ver la estancia media en días según el sexo de nuestros pacientes, observándose como el hecho de ser hombre o mujer influye en el sentido de

Tabla I

Número de ingresos según diagnósticos psiquiátricos reagrupados CIE-9/CIE-10 de pacientes ingresados desde 1975 a 2000 (26 años)

\begin{tabular}{lccc} 
Diagnósticos & Media de episodios de Ingreso & Desviación estándar & N. ${ }^{\circ}$ de Casos \\
\hline Demencias & 1,8128 & 2,5642 & 219 \\
Psicosis Orgánicas Transitorias & 1,9855 & 2,0433 & 138 \\
Psicosis Orgánicas & 2,5135 & 3,397 & 74 \\
Psicosis Alcohólicas & 1,689 & 1,6851 & 254 \\
Dependencia de Drogas & 1,6763 & 1,5168 & 346 \\
Dependencia a Alcohol & 1,8867 & 1,8778 & 450 \\
Psicosis Esquizofrénicas & 2,5954 & 2,6947 & 2153 \\
Trast. Paranoides Crónicos & 1,6558 & 1,298 & 337 \\
Psicosis Agudas & 1,4769 & 1,016 & 497 \\
Trast. Esquizoafectivos & 4,7727 & 3,9444 & 396 \\
Trast. Neuróticoss & 2,139 & 2,5936 & 403 \\
Trast. Personalidad & 2,7893 & 3,3122 & 655 \\
Trast. Sexuales & 1,3333 & 0,5774 & 3 \\
Disfunciones Fisiológicas & 1,8056 & 1,4106 & 36 \\
Anorexias & 2,531 & 2,6116 & 145 \\
Oligofrenias & 1,6961 & 1,572 & 102 \\
Trast. Emocionales Infancia & 5,6296 & 5,4076 & 27 \\
Trast. Adaptación & 1,6284 & 1,5933 & 218 \\
Psicosis Maníacas & 3,9964 & 5,3155 & 831 \\
Psicosis Depresivas & 4,2532 & 6,5943 & 1390 \\
Otras Psicosis Maniacodepresivas & 4,7655 & 5,5578 & 145 \\
Distimias & 2,256 & 2,3663 & 586
\end{tabular}

El número medio de episodios de ingreso fue de 2,8215 con una desviación estandar de 3,8967.

Departamento de Psiquiatría (Prof. A. Seva). Hospital Clínico Universitario. Zaragoza.

Tabla II

Estancia media según sexo de pacientes ingresados desde 1975 a 2000 (26 años)

\begin{tabular}{lccc} 
& n. $^{\circ}$ de días de media & Desviación / estándar & N. $^{\text {o de Casos }}$ \\
\hline Hombre & 18,8448 & 20,8931 & 5590 \\
Mujer & 22,6452 & 22,4214 & 5365
\end{tabular}

La estancia media para el total de la población es de 20,7077 días con una desviación estándar de 21,7379. Departamento de Psiquiatría (Prof. A. Seva). Hospital Clínico Universitario. Zaragoza. 
la mayor probabilidad que ellas tienen de permanecer mas días hospitalizadas (23 días frente a 19 días los hombres).

En la tabla III figuran las estancias medias en días según las edades de nuestros enfermos en el momento del ingreso, pudiendo observarse como las estancias medias mas prolongadas las alcanzan los pacientes con edades comprendidas entre 60-69 años, siguiéndoles los que tienen entre 50-59 y 70-79 años. Sorprende las bajas estancias medias de los mayores de 80 años.

En la tabla IV aparecen las estancias medias según la actividad laboral de los pacientes, observándose como la situación de Inactivo, en dónde se acumulan muchas amas de casa, ofrecen las estancias medias mas prolongadas, siendo por otro lado, las de los marginados las mas bajas.

En la tabla V figura la estancia media según el estado civil de los pacientes,

Tabla III

Estancia media según edades de pacientes ingresados desde 1975 a 2000 (26 años)

\begin{tabular}{cccc} 
& Media de días & Desviación / estandar & N. $^{\text {o de casos }}$ \\
\hline $10-19$ & 19,8615 & 21,6613 & 1126 \\
$20-29$ & 18,4362 & 20,3965 & 2549 \\
$30-39$ & 18,7174 & 24,5873 & 2265 \\
$40-49$ & 19,2634 & 17,1393 & 1841 \\
$50-59$ & 24,4356 & 24,1101 & 1451 \\
$60-69$ & 27,7015 & 21,4766 & 1042 \\
$70-79$ & 24,263 & 18,5274 & 384 \\
$80-89$ & 18,6111 & 14,8992 & 72 \\
$90-99$ & 7,6667 & 1,5275 & 3 \\
\hline
\end{tabular}

La estancia media para el total de la población es de 20,7042 días con una desviación estándar de 21,7371.

Departamento de Psiquiatría (Prof. A. Seva). Hospital Clínico Universitario. Zaragoza.

Tabla IV

Estancia media según actividad profesional de pacientes ingresados desde 1975 a 2000 (26 años)

\begin{tabular}{lccc} 
& Media de días & Desviación / estándar & N. ${ }^{\circ}$ de casos \\
\hline Activo cuenta propia & 18,5536 & 14,8506 & 271 \\
Activo cuenta ajena & 17,6614 & 18,45 & 2950 \\
Inactivo & 21,8912 & 21,0531 & 7689 \\
Marginado & 14,2791 & 20,8456 & 64 \\
\hline
\end{tabular}

La estancia media para el total de la población es de 20,7533 días con una desviación estándar de 21,7465.

Departamento de Psiquiatría (Prof. A. Seva). Hospital Clínico Universitario. Zaragoza.

Tabla V

Estancia Media segun Estado Civil de pacientes ingresados desde 1975 a 2000 (26 años)

\begin{tabular}{lccc} 
& Media de días & Desviación / estándar & N. ${ }^{\circ}$ de casos \\
\hline Soltero & 19,5593 & 19,37702 & 5143 \\
Casado & 21,3259 & 21,103 & 4771 \\
Viudo & 24,4846 & 19,9005 & 554 \\
Religioso & 21,2 & 13,9427 & 12 \\
Separado/divorciado & 19,1703 & 22,9557 & 494 \\
\hline
\end{tabular}

La estancia media para el total de la población es de 20,7514 días con una desviación estándar de 21,7534. Departamento de Psiquiatría (Prof. A. Seva). Hospital Clínico Universitario. Zaragoza. 
pudiendo verse como la situación de viudedad representa unas estancias medias elevadas de unos 24 días, probablemente y entre otras cosas debido a la mayor presencia de mujeres amas de casa en este grupo.

En la tabla VI podemos ver las diferencias de las estancias medias según el domicilio de residencia de nuestros pacientes. Observamos como Teruel, Huesca y Logroño presentan las mas elevadas, siendo curio- samente las mas bajas, las que aportan los pacientes procedentes de otras provincias españolas y del extranjero.

En la tabla VII vemos el reflejo de estas estancias medias según la clase social de pertenencia de los enfermos.

En la tabla VIII observaremos ver las grandes diferencias existentes, según los ingresos se hayan producido de forma voluntaria, compulsiva o siguiendo una orden judicial.

\section{Tabla VI}

Estancia media según lugar de residencia de pacientes ingresados desde 1975 a 2000 (26 años)

\begin{tabular}{lccc} 
& N. $^{\circ}$ de días de media & Desviación / estándar & N. ${ }^{\circ}$ de casos \\
\hline Zaragoza & 20,3718 & 20,7822 & 10105 \\
Huesca & 24,7268 & 22,9952 & 209 \\
Teruel & 24,51 & 18,2944 & 271 \\
Soria & 20,3953 & 17,6583 & 46 \\
Logroño & 24,898 & 20,6138 & 52 \\
Navarra & 10,6563 & 13,6207 & 34 \\
Otras provincias & 14,3665 & 25,8402 & 209 \\
Extranjero & 17,2045 & 24,9143 & 48
\end{tabular}

La estancia media para el total de la población es dee 20,7683 días con una desviación estándar de 21,7551. Departamento de Psiquiatría (Prof. A. Seva), Hospital Clínico Universitario. Zaragoza.

Tabla VII

Estancia media según clase social de pacientes ingresados desde 1975 to 2000 (26 years)

\begin{tabular}{lccc} 
& N. $^{\text {o de días de media }}$ & Desviación / estándar & N. $^{\text {o de casos }}$ \\
\hline Alta & 21,4545 & 17,2867 & 26 \\
Media-alta & 22,4759 & 23,0582 & 176 \\
Media & 20,7229 & 20,7423 & 8165 \\
Media-baja & 21,4089 & 20,3779 & 1804 \\
baja & 18,2215 & 14,8908 & 557 \\
Marginal & 18,1531 & 15,2894 & 246 \\
\hline
\end{tabular}

La estancia media para el total de la población es de 20,7683 días con una desviación estándar de 21,7551. Departamento de Psiquiatría (Prof. A. Seva), Hospital Clínico Universitario. Zaragoza.

Tabla VIII

Estancia media según forma de ingreso de pacientes ingresados desde 1975 a 2000 (26 años)

\begin{tabular}{lccc} 
& N. ${ }^{\circ}$ de días de media & Desviación / estándar & N. ${ }^{\circ}$ de casos \\
\hline Normal & 20,7256 & 20,4547 & 10715 \\
Judicial & 42,1963 & 60,5412 & 110 \\
Compulsivo & 29,483 & 34,4032 & 149 \\
\hline
\end{tabular}

La estancia media para el total de la población es de 21,0878 días con una desviación estandar de 21,6881. Departamento de Psiquiatría (Prof. A. Seva), Hospital Clínico Universitario. Zaragoza. 
Con gran diferencia son los ingresos por orden judicial los que presentan unas estancias medias mas prolongadas con 42 días, seguidos por los ingresos compulsivos con unas estancias medias de 29 días.

En la figura 10 podemos ver los porcentajes de días de hospitalización, pudiendo observarse como la tercera parte de nuestros ingresos consumieron menos de 10 días de estancia. Por otro lado, un $17 \%$ de las hospitalizaciones habidas consumieron entre $11 \mathrm{y}$ 15 días. Ello quiere decir que la mitad de nuestras hospitalizaciones, a lo largo de estos 25 años, consumieron menos de 15 días en el hospital. El 81\% de los 10.971 ingresos analizados no sobrepasaron el mes de estancia media con lo que se situaron por debajo del estándar medio propio de las Unidades de Agudos de los hospitales generales (30 días).

A pesar de ello, el 19\% restante consumió mas de 31 días. Un grupo menor que representa el $4 \%$ de los ingresos superó los 2 meses, e incluso el $0,2 \%$ de los casos per- maneció en nuestro Servicio por un periodo superior a medio año. Razones del tipo de ingresos por orden judicial o la falta absoluta de recursos asistenciales para hospitalizaciones de media y larga estancia en el entorno, son las responsables de estas situaciones tan negativas para los enfermos que han de sufrir estos largos periodos de hospitalización en las Unidades de Agudos, que por otro lado no estan preparadas para ellos ni estructural ni funcionalmente.

En la figura 11 hemos representado los tiempos de hospitalización por diagnósticos reagrupados CIE-9/CIE-10 a lo largo de estos 26 años.

Puede observarse el peso relativo que las estancias, agrupadas en diferentes intervalos, tienen para cada patología psiquiátrica. Así, en amarillo aparecen las mas cortas con los Trastornos de Adaptación a la cabeza, siguiéndoles los Trastornos Emocionales en la Infancia y Adolescencia, los Trastornos de Personalidad, las Dependencias a

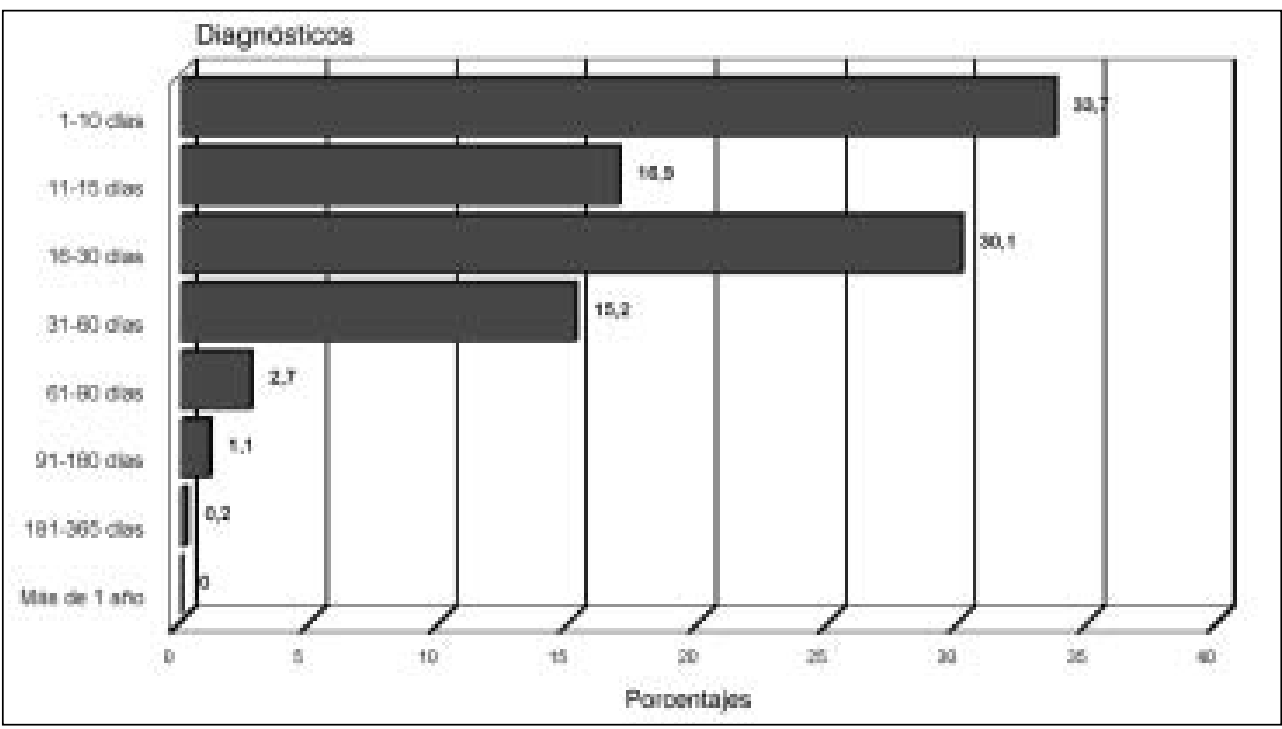

Figura 10. Porcentajes de días de hospitalización producidos por los pacientes ingresados desde 1975 a 2000. Departamento de Psiquiatría (Prof. A. Seva). Hospital Clínico Universitario. Zaragoza. 


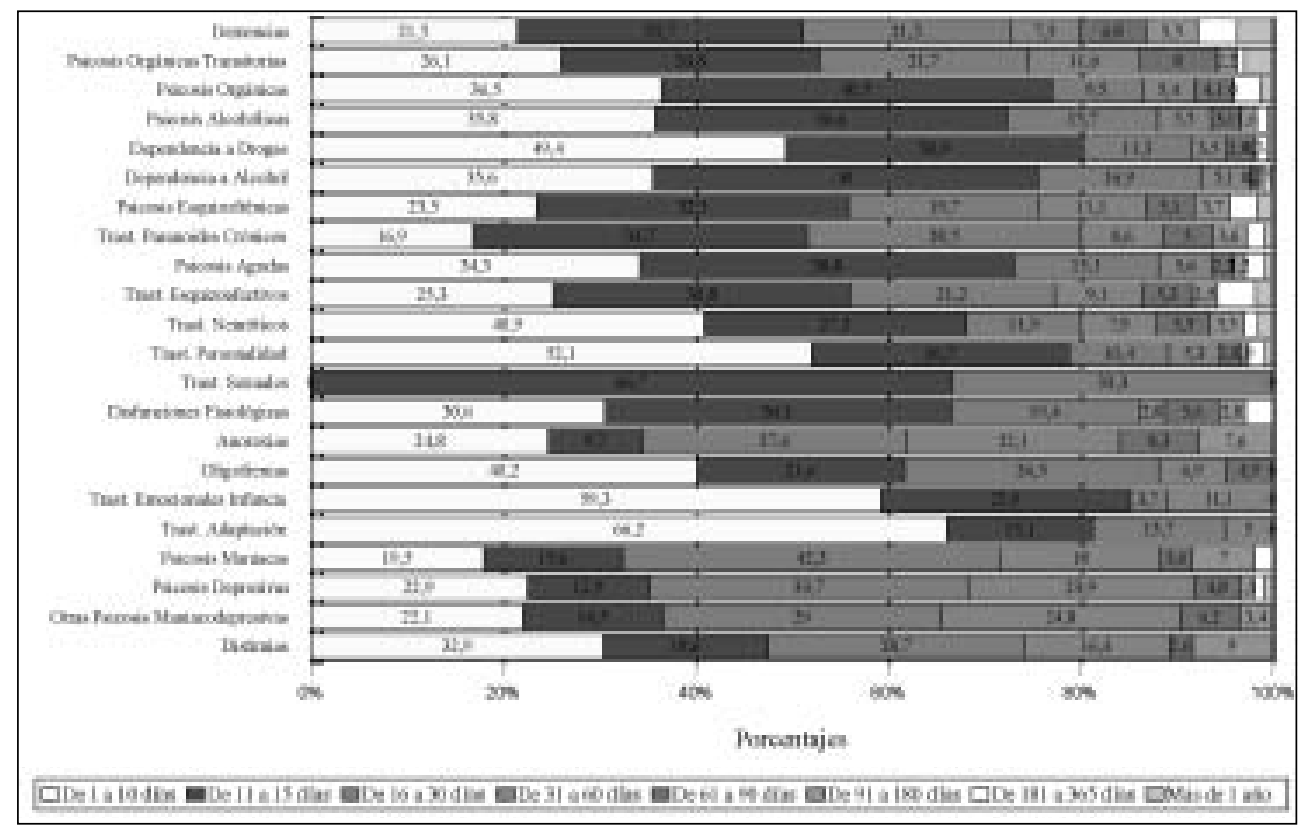

Figura 11. Tiempos de hospitalización por diagnósticos reagrupados CIE-9/CIE-10. Pacientes ingresados a lo largo de 26 años (1975 a 2000).

Departamento de Psiquiatría (Prof. A. Seva). Hospital Clínico Universitario. Zaragoza.

Drogas y los Trastornos Neuróticos. Las proporciones de tiempos aparecen muy claramente para cada uno de los diagnósticos, como es fácil observarlo.

En la tabla IX podemos ver las estancias medias depuradas para la construcción de los GRDs, en este caso habiendo reagrupado los diagnósticos CIE-9 y CIE-10.

\section{Discusión}

Con todos estos datos en la mano podemos, en líneas generales, hacer una previsión de la duración de la estancia de un determinado paciente que ingresa en el Servicio, simplemente conociendo su diagnóstico al ingreso, su historia anterior de reingresos, su sexo de pertenencia, su edad, su estado civil, su actividad profesional, su clase social, su domicilio de residencia, el tipo de ingreso con el que llega, su pertenencia asistencial y por supuesto la comorbilidad existente.

Los G.R.D. (Grupos Relacionados con el Diagnóstico) y los Case-Mix, que permiten conocer el nivel de complejidad de la patología asistida, son muy diferentes en el campo de la Psiquiatría como hemos tenido ocasión de ver en páginas anteriores.

Una muestra de los defectos estructurales propios de los GRDs psiquiátricos los tenemos en la distribución de las Altas por GRD que fueron en el año 2000 en nuestro Departamento Psiquiátrico (tabla X).

Las autoridades sanitarias no deberían así seguir asignando GRDs tan inespecíficos como el de Psicosis en donde pueden incluirse una gran cantidad de clases diagnósticas absolutamente inespecíficas. Del 
Tabla IX

Estancias medias en días depuradas para la construcción de los G.R.D. (Integración de CIE-9/CIE-10) de pacientes ingresados desde 1975 a 2000 (26 años)

\begin{tabular}{lccc} 
Diagnosis & N. $^{\circ}$ de días de media & Desviación estandar & N. ${ }^{\circ}$ de casos \\
\hline Demencias & 26,6027 & 26,8476 & 219 \\
Psicosis orgánicas transitorias & 25,4275 & 37,5383 & 138 \\
Psicosis orgánicas & 16,5541 & 17,8687 & 74 \\
Psicosis alcohólicas & 16,5315 & 21,7562 & 254 \\
Dependencia a drogas & 13,3699 & 15,2869 & 346 \\
Dependencia a alcohol & 15,0289 & 18,1076 & 450 \\
Psicosis esquizofrénicas & 22,7952 & 23,1266 & 2153 \\
Trast. paranoides crónicas & 21,7923 & 16,3639 & 337 \\
Psicosis agudas y psicógenas & 16,8414 & 15,4367 & 498 \\
Trast. esquizoafectivos & 22,5934 & 22,3089 & 396 \\
Trast. Neuróticos & 18,1241 & 18,9954 & 403 \\
Trast. personalidad & 14,7191 & 24,061 & 655 \\
Trast. sexuales & 16,6667 & 4,0415 & 3 \\
Disfunciones fisiológicas & 17,6944 & 14,9739 & 36 \\
Anorexias & 33 & 29,3915 & 145 \\
Oligofrenias & 16,6961 & 15,7328 & 102 \\
Trast. emocionales y compto. inf./juv. & 12,2593 & 11,9794 & 27 \\
Trast. adaptación y estrés & 9,8037 & 9,0078 & 219 \\
Psicósis maniacas & 23,899 & 19,9019 & 832 \\
Psicosis depresivas & 26,1774 & 24,9646 & 1392 \\
Otras psicosis maniacodepresivas & 27,9034 & 23,5918 & 145 \\
Distimias & 20,0222 & 17,6459 & 586 \\
\hline Desastan
\end{tabular}

Departamento de Psiquiatría (Prof. A. Seva). Hospital Clínico Universitario. Zaragoza.

Tabla X

\begin{tabular}{lcccc} 
GRDs & Número & E. Media & E. Media depurada & Peso relativo \\
\hline Psicosis & 248 & 16,2 & 13,2 & 1,5274 \\
Neurosis Depresiva & 21 & 10,1 & 9,2 & 0,7557 \\
Otras Neurosis & 27 & 13,9 & 14,8 & 1,0537 \\
Trastornos Personalidad & 30 & 13,6 & 10,4 & 0,6880 \\
Abuso/Dependencia Alcohol & 19 & 12,7 & 11,3 & 0,5733 \\
Reacción Aguda & 15 & 13,1 & 7,7 & 0,7797 \\
Intoxicación & 11 & 9,5 & 5,4 & 0,5372 \\
\hline
\end{tabular}

mismo modo los otros GRDs que como Otras neurosis, Neurosis Depresiva, Trastornos Personalidad, Abuso/Dependencia Alcohol, Reacción Aguda e Intoxicación, son de una pobreza extraordinaria en el momento actual de desarrollo de nuestra especialidad. El problema de la aplicación de los GRDs en Psiquiatría preocupa tanto en España que ya en el año 1998 tuvo lugar en Sevilla una Reunión de Expertos bajo el título de Los DRGs en Psiquiatría.

Confiamos en que progresivamente y a partir de una mejor definición y especificación de las patologías psiquiátricas, sea mediante sistemas como los GRDs u otros, 
iremos construyendo medidas de eficiencia y de eficacia que nos permitan, incluso llegar a calcular con una gran exactitud los costes por proceso en Psiquiatría.

\section{Reconocimiento}

Este trabajo pertenece a un Proyecto de Investigación financiado por el FIS PI021501.

\section{Bibliografía}

ASENJO, M.A. Las Claves de la Gestión Hospitalaria, Barcelona, Ediciones Gestion 2000 S.A., 1999.

AVERILL, R.F., GOLDFIELD, N., STEINBECK, B.A. Grupos Relacionados por el Diagnóstico (GRDs). Sigesa, 1995.

BEN-TOVIM, D., ELZINGA, R., PILLA, J., MCALLISTER, S., WILHEIM, K., LIPTON, G., POLS, R., FRANKLIN, J., WATERS, M.M. A case-mix for mental health services: The development of the mental health and substance abuse components of the Australian national diagnosis-related groups. Australian and New Zealand Journal of Psychiatry, 30, 4, 450-456, 1996.

BEN-TOVIM, D.I., ELZINGA, R.H. Making case-mix work for Psychiatry. Medical Journal of Australia, 161 (Suppl.), 533-536, 1994.

BOOT, B., MAY, W., ANDREWS, G. Disability, outcome and case-mix in acute psychiatric in-patient units. British Journal of Psychiatry, 171, 242-246, 1997.

CASAS, M. GRD. Una guía práctica para médicos. Cuadernos de Gestión clínica, Iasist S.A., 1995.

ERRASTI, F. Principios de Gestión Sanitaria, Madrid, Díaz de Santos, 1996.
GOLDBERG, R.J., DALY, D., BACKSTROM, D. Psychiatric Complications and Comorbidities in Medical Inpatients: The Inadequacy of Attestation at Discharge. General Hospital Psychiatry, 18, 2, 102-105, 1996.

GOLDNEY, R.D., ELZINGA, R.H., KENT, P. Casemix and Affective Disorders: A Comparison of Private and Public Psychiatric Systems. Australians and NewZealand Journal of Psychiatry, 30, 4, 511-515, 1996.

HUNTER, C.E., MCFARLANE, A.C. DRGs and Australian Psychiatry. Australian and New Zealand Journal of Psychiatry, 28, 1, 114-120, 1994.

KOVESS, V., SOYRIS, D. The introduction of medical data into hospitals data base and the prospective payment in Psychiatry: Feasibility and consequences. Léncephale, 20, 1, 37-45, 1994.

MEISE, U., HINTERHUBER, H. Inpatient Coverage Based on Diagnosis-related Groups (LKF) Financial Losses for Psychiatry? Neuropsychiatrie, 12, 4, 177-186, 1998.

PIRZADA, S.R., RIES, R., LOGERFO, J.P. Cost of Comorbid Alcohol and Drug Problems. American Journal on Addictions, 6, 3, 193-204, 1997.

Reunión sobre Los GRD en Psiquiatría. 23-24 de Abril, Sevilla, 1998.

STOSKOPF, C., HORN, S.D. Predicting length of stay for patients with psychoses. Health Services Research, 26, 6, 743-766, 1992.

TEMES MONTES, J.L., PASTOR ALDEGUER, V., DÍAZ FERNÁNDEZ, J.L. Manual de Gestión Hospitalaria, Madrid, McGraw-Hill-Interamericana S.A., 1997.

Dirección para correspondencia:

A. Seva

Catedrático de Psiquiatría y Psicología Médica, Jefe del Departamento de Psiquiatría, Facultad de Medicina y Zaragoza Clinico Universitario

Avd. San Juan Bosco

50009 Zaragoza

ESPAÑA 
